\title{
Pollution des eaux superficielles par les produits phytosanitaires : de la recherche appliquée aux premiers résultats sur les bassins du programme Bretagne Eau Pure
}

\author{
Surface water pollution by pesticides : from applied research to the first \\ results on Bretagne Eau Pure program basins
}

par H. Gillet

Direction Régionale de l’Agriculture et de la Forêt de Bretagne

Service Régional de la Protection des Végétaux.

In Brittany, the reconquest of water quality represents a major challenge for which political and economical managers join their efforts and particularly within the Bretagne Eau Pure program that sets every effort to initiate this reconquest, notably concerning pesticides, within 5 years.

Locally, the approach to such a form of pollution was made originally with the creation by the Region Prefect at the end of 1990 of a consultation and interdisciplinary coordination Cell taken in charge by the DRAF.

The applied research studies achieved for several years within this Cell enabled us to make a first diagnostic and to determine orientations in order to take action in the Bretagne Eau Pure program basins where the objectives of reconquest of the rivers' water quality corresponding to statutory concentrations in alimentation waters, had been set up.

An action method based on the adaptation of different ways of weeding, taking into account the risk assigned to the fields and zones that have to be weeded, has been developed in order to reach these objectives.

The application of this procedure has already provided some first encouraging results towards water quality improvement.

\section{I INTRODUCTION}

La prise en compte de la pollution des eaux par les produits phytosanitaires est relativement récente puisqu'elle n'intervient qu’avec le décret du 3 janvier 1989 relatif aux eaux destinées à la consommation humaine qui introduit les pesticides et produits apparentés dans les paramètres soumis à vérification. En Bretagne, l'approche de cette nouvelle forme de pollution s'est faite de manière coordonnée par la mise en place en 1990 par le préfet de Région d'une cellule technique pluridisciplinaire : la CORPEP (Cellule d'Orientation Régionale pour la Protection des Eaux contre les Pesticides) $|5|$.

Cette cellule régionale composée d'organismes scientifiques et universitaires, des services de l'Etat, de l'agence de l'eau, des collectivités territoriales et des professionnels a réussi à créer une dynamique de groupe qui a permis chaque année de définir des priorités d'études dont les premiers résultats qui ont été publiés au cours d'un colloque organisé à Rennes le 27 novembre 1995 ont permis de dégager des orientations pour agir |3|.

Les vingt bassins versants de démonstration et d'action renforcée retenus dans le cadre du programme Bretagne Eau Pure II ont été choisis comme support pour la mise en œuvre de programmes de reconquête de la qualité de l'eau notamment en matière de nitrates et de pesticides. Sur chaque bassin, le programme fait l'objet d'un contrat pour cinq ans établi par une structure porteuse (syndicat ou association) regroupant tous les acteurs locaux en liaison avec les partenaires de Bretagne Eau Pure II. Ces contrats se basent sur l'exigence de résultats par rapport à des objectifs quantifiés de reconquête de la qualité des eaux. En matière de produits phytosanitaires, les objectifs fixés pour les eaux brutes correspondent aux valeurs paramétriques pour l'eau potable.

Afin de mettre rapidement au service de ces actions de terrain les résultats de la recherche et des expérimentations de la CORPEP, il a été mis en place une Cellule Phytosanitaire au sein du programme Bretagne Eau Pure (Cellule Phyt'eau) dont le rôle est de définir, structurer et développer des stratégies de prévention des pollutions.

La méthodologie de diagnostic et d'actions mise en oeuvre ainsi que les premiers résultats sont présentés dans ce document. 
II $\square$ LES ACQUIS DE LA RECHERCHE TRADUITS EN MÉTHODOLOGIE DE DIAGNOSTIC ET D'ACTION SUR LES BASSINS VERSANTS

\subsection{Rappel sur les principaux acquis de la recherche}

Les travaux de la CORPEP ont mis en évidence le fait que la contamination des eaux superficielles, ressource principale dans l'ouest pour la production d'eau potable, trouve son origine dans les pratiques de désherbage qu'elles soient agricoles ou non agricoles (communes, administrations, particuliers) [6]. Ils ont également mis en lumière que le risque de contamination des eaux dépendait à la fois de critères propres aux substances actives (KOC, hydrosolubilité et persistance) et de leurs applications sur des parcelles (agricoles) ou zones (non-agricoles) particulières à partir desquelles les transferts étaient très importants [7].

La prise en compte de ces parcelles ou zones à risque apparaît donc comme prioritaire pour la conduite d'actions de reconquête de la qualité des eaux. L'enjeu est donc de les localiser à la fois à l'échelle du bassin versant, de chaque exploitation agricole, dans les collectivités et chez les particuliers pour y appliquer des itinéraires techniques spécifiques non polluants couplés à des aménagements fonciers.

\subsection{Une méthodologie de diagnostic et d'action innovante appliquée à l'ensemble des bassins de Bretagne Eau Pure}

\subsection{Volet agricole}

Cette méthodologie est basée sur le repérage des sous-bassins à risque et sur le classement des parcelles à l'intérieur des sous-bassins à problème.

Elle repose sur la réalisation tout au long de l'année de campagnes de dosages immuno-enzymatiques des résidus de triazines sur un grand nombre de points de prélèvements. Cet échantillonnage à grande échelle est rendu possible grâce au coût réduit de ces tests par rapport aux analyses classiques par chromatographie [8].

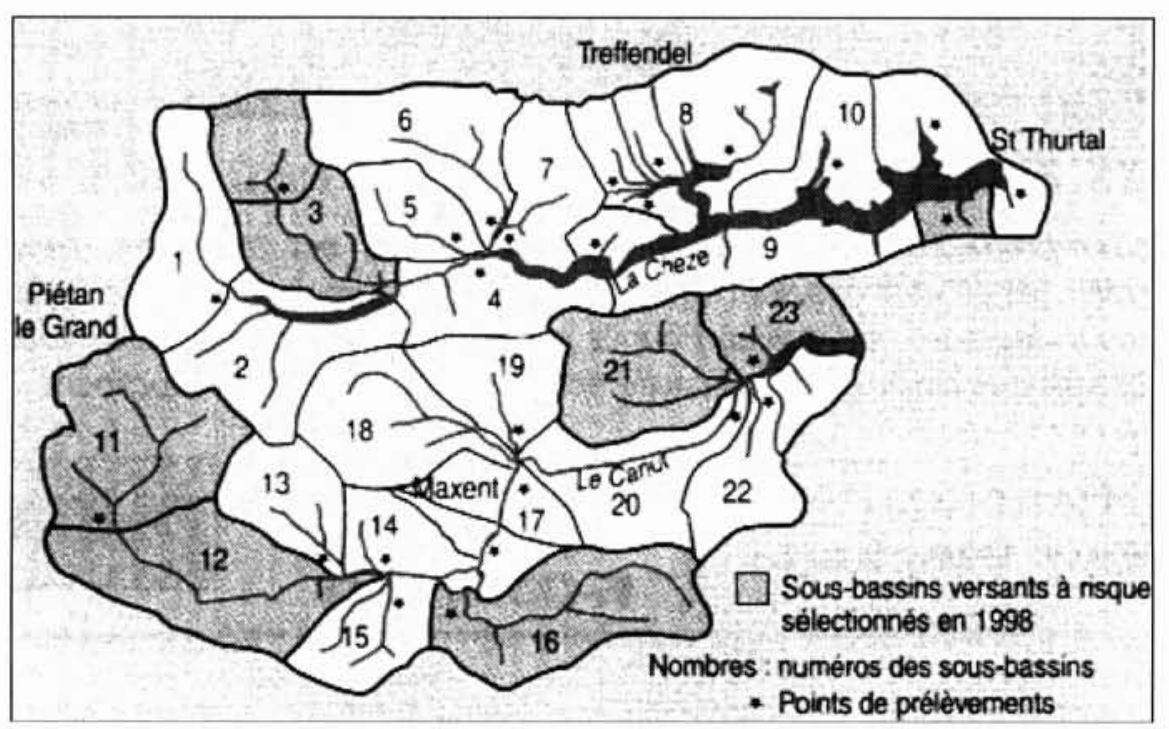

1. Bassin Versant Cheze Canut.
Les résultats obtenus sur la plupart des bassins versants montrent l'intérêt de la démarche qui débouche sur un zonage des actions laissant entrevoir une reconquête plus rapide et moins coûteuse de la qualité de l'eau. A titre d'exemple la figure 1 montre le bilan des campagnes de dosages immuno-enzymatiques des triazines sur les sousbassins de la Chèze et du Canut. Sur les vingt-trois sous-bassins répertoriés au total, six font l'objet de dépassements quasi permanents des objectifs du programme Bretagne Eau Pure.

Après cette étape de repérage des sous-bassins à risque, un classement des parcelles à risque est réalisé dans chaque exploitation située dans les sous-bassins à problème. Pour réaliser ce classement, une méthode a été finalisée par la commission transfert de la CORPEP. Cinq facteurs de risque ont été retenus et hiérarchisés. Le tableau 1 les présente de façon synthétique par ordre d'importance [1]. Leur traduction en un critère y est définie. Selon le cas, deux ou trois classes ont été choisies par critère. La méthode SIRIS, méthode multicritère permettant la hiérarchisation des facteurs, a été retenue comme outil de classement [9].

Sur le terrain, des tables (Cf. Tableau 2) reprenant le rangement SIRIS sont utilisées pour réaliser le classement des parcelles. Ce classement prend en compte les cinq facteurs et les classes définies ci-dessus, soit en théorie 108 combinaisons. Les rangs SIRIS, notés au maximum sur 115 , sont ramenés sur ces tables à un rangement de 0 à 100 pour faciliter la lisibilité du classement.

Pour ce classement on définit sur le terrain en présence de l'agriculteur les parcelles élémentaires à partir de l'occupation du sol et du sens des écoulements. Par exemple, deux parcelles jointives ayant la même occupation du sol seront séparées si une discontinuité en terme d'écoulement est visible sur le terrain. A l'opposé, une même parcelle en terme d'occupation du sol peut être découpée en deux si elle présente des écoulements divergents conduisant à des portions différentes du réseau hydrographique.

A l'issue de la visite de terrain et du classement, il est remis à chaque exploitant un plan sur lequel sont figurés les niveaux de risque de chacune des parcelles. Un code en trois couleurs a été adopté pour matérialiser les différents niveaux de risque.

Ce classement des parcelles constitue un des objectifs essentiels de réduction de la pollution diffuse des eaux superficielles. Il a été conçu comme un outil pédagogique destiné aux agriculteurs, outil qui devrait les inciter à la mise en cuvre des mesures agri-environnementales et/ou d'aménagements destinés à lutter contre l'érosion et de surcroît à limiter le surcoût de certains programmes spécifiques de désherbage préconisés sur les parcelles à risque.

Toujours par souci d'harmonisation de la démarche sur l'ensemble des bassins du programme, il a été mis en place pour les personnes chargées de la mise en œuvre de ce classement sur le terrain, une session de formation.

Parallèlement à l'élaboration d'une méthode de classement des parcelles, des propositions d'itiné- 


\begin{tabular}{|c|c|c|c|}
\hline FACTEUR & CRITERE & DEFINITION ANNEXE & CLASSES \\
\hline 1-Distance & $\begin{array}{l}\text { Distance entre le point le plus aval } \\
\text { de la parcelle traitée et le cours } \\
\text { d'eau circulant }\end{array}$ & $\begin{array}{c}\text { Le cours d'eau est circulant } \\
\text { quand l'cau y coule de façon } \\
\text { continue durant la période } \\
\text { hivernale }\end{array}$ & $\begin{array}{l}<20 \mathrm{~m} / 20 \text { classes: } \\
200 \mathrm{~m}\end{array}$ \\
\hline 2-Pente & $\begin{array}{l}\text { Pente moyenne sur l'ensemble de } \\
\text { la parcelle traitée }\end{array}$ & & $<3 \% \frac{3 \text { classes: }}{/ 3 \text { à } 5 \% />5 \%}$ \\
\hline 3-Drainage & $\begin{array}{l}\text { Drainage agricole souterrain de la } \\
\text { parcelle }\end{array}$ & & $\begin{array}{l}2 \text { classes: } \\
\text { présence / absence }\end{array}$ \\
\hline 4-Longueur & $\begin{array}{l}\text { Longueur maximale de la parcelle } \\
\text { dans le sens des écoulements }\end{array}$ & & $\begin{array}{l}3 \text { classes: } \\
<50 \mathrm{~m} / 50 \text { à } 150 \mathrm{~m} /> \\
150 \mathrm{~m}\end{array}$ \\
\hline $\begin{array}{l}\text { 5-Protection } \\
\text { avale }\end{array}$ & $\begin{array}{l}\text { Présence d'une protection continue } \\
\text { et durable à l'aval de la parcelle, } \\
\text { empêchant tout transfert direct }\end{array}$ & $\begin{array}{l}\text { Correspond soit à un dispositif } \\
\text { enherbé d'au moins } 5 \text { ans, un } \\
\text { boisement d'au moins } 20 \mathrm{~m} \text { ou } \\
\text { à un dispositif haie/talus }\end{array}$ & $\begin{array}{l}\frac{2 \text { classes : }}{\text { présence / absence }} \\
\text { lat }\end{array}$ \\
\hline
\end{tabular}

Tableau I - Facteurs de risques.

\begin{tabular}{|c|c|c|c|c|c|c|c|c|c|c|}
\hline \multirow{2}{*}{\multicolumn{2}{|c|}{ Parcelle drainée }} & \multicolumn{9}{|c|}{ Distance } \\
\hline & & \multicolumn{3}{|c|}{$>200 \mathrm{~m}$} & \multicolumn{3}{|c|}{20 à $200 \mathrm{~m}$} & \multicolumn{3}{|c|}{$<20 \mathrm{~m}$} \\
\hline $\begin{array}{c}\text { protection } \\
\text { aval }\end{array}$ & $\begin{array}{l}\text { longueur } \\
\text { parcelle }\end{array}$ & $<3 \%$ & $\begin{array}{l}\text { pente } \\
3 \text { à } 5 \%\end{array}$ & $>5 \%$ & $<3 \%$ & $\begin{array}{c}\text { pente } \\
3 \text { a } 5 \%\end{array}$ & $>5 \%$ & $<3 \%$ & $\begin{array}{c}\text { pente } \\
3 \text { à } 5 \%\end{array}$ & $>5 \%$ \\
\hline \multirow{3}{*}{ présence } & $<50 \mathrm{~m}$ & 6 & 13 & 20 & 22 & 31 & 41 & 38 & 50 & 632 \\
\hline & \begin{tabular}{|l|}
50 à $150 \mathrm{~m}$ \\
\end{tabular} & 9 & 17 & 24 & 27 & 37 & 48 & 46 & 59 & $\overline{2}$ \\
\hline & $>150 \mathrm{~m}$ & 11 & 20 & 29 & 32 & 43 & 5823 & 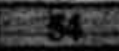 & 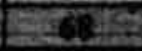 & Ex \\
\hline \multirow{3}{*}{ absence } & $<50 \mathrm{~m}$ & 9 & 17 & 26 & 30 & 41 & 5 & 51 & 65 & 79 \\
\hline & 50 à $150 \mathrm{~m}$ & 11 & 20 & 29 & 36 & 48 & 50 & 60 & 275 & $5 \times 0$ \\
\hline & $>150 \mathrm{~m}$ & 16 & 26 & 37 & 42 & $58 x-1$ & 5835 & $\operatorname{rrs} 60=$ & Exises: & 2100 \\
\hline
\end{tabular}

Tableau 2 - Tables de classement des parcelles.

raires de désherbage adaptés à chaque niveau de risque parcellaire ont été élaborées par la commission transfert de la CORPEP

Les désherbants utilisés sur maïs et céréales ont été classés en trois groupes suivant leur comportement de mobilité et de persistance dans les sols ainsi qu'en fonction de la dose apportée à l'hectare. Le classement réalisé n’a pas pour objectif de procéder à une homologation bis, mais d'aboutir au contraire à une meilleure lisibilité et compréhension de ces nouveaux concepts par les agriculteurs.

Le groupe I par exemple. dans lequel sont classées les substances présentant un risque limité de pollution des eaux superficielles. (ex : Sulfonylurées) se définit par les critères suivants :

dose $/$ hectare $/$ an $<=500$ grammes .

ou $\mathrm{KOC}>1000$

ou DT $50<8$ jours

A l'opposé. le groupe III qui compte les substances à plus haut risque (ex : atrazine) a été défini de la manière suivante :

dose/hectare/ $>500$ grammes

et $\mathrm{KOC}<1000$

et DT $50>30$ jours.
Une correspondance entre ces groupes de produits et les niveaux de risque parcellaire a été proposée et figure dans le tableau 3.

Sur les bassins versants, ces préconisations spécifiques sont consignées dans des chartes phytosanitaires qui sont élaborées en concertation avec l'ensemble des prescripteurs intervenant sur le terrain.

A côté de ces préconisations spécifiques en matière de substitution, d'autres solutions techniques sont proposées pour les parcelles à risque. Elles sont présentées dans la suite de ce document.

\subsubsection{Volet non agricole}

\section{Un plan de désherbage pour les collectivités.}

Le classement des zones à risque pour le secteur non agricole n'est pas aussi élaboré que pour le secteur agricole. Néanmoins, pour les communes situées sur les bassins versants du programme Bretagne Eau Pure, des plans de désherbage communaux sont mis en place. Ces plans permettent de distinguer pour les surfaces désherbées (comme pour les parcelles agricoles) trois niveaux de risque définis par rapport à leur degré d'imperméabilisation, leur pente (ruissellement) et leur localisation par rapport à la ressource en eau. 


\begin{tabular}{|c|c|c|c|}
\hline PARCELLE & FAIBLE & MOYEN & ELEVE \\
\hline GROUPE DE PRODUITS & & & oui \\
\hline 1 & oui & oui & non \\
\hline 2 & oui & oui & $\begin{array}{c}\text { ou } \\
(\text { sauf IPU }<\text { ou }=500 \mathrm{~g})\end{array}$ \\
\hline 3 & oui & $\begin{array}{c}\text { non } \\
\text { (sauf atrazine }<\text { ou }=500 \mathrm{~g})\end{array}$ & . \\
\hline
\end{tabular}

Tableau 3. - Correspondance groupes de produits / niveaux de risque.

Sur les zones à risque faible et/ou moyen dont les surfaces ont été recensées au moment de la réalisation de ce plan, un choix limité de substances est proposé aux communes.

$\mathrm{Si}$ les préconisations en matière de désherbage sont moins bien maîtrisées que pour le maïs ou les céréales, faute de données sur les transferts de ces produits en milieu urbain, il n'en demeure pas moins que la liste des produits proposée actuellement aux communes et élaborée à partir des doses homologuées/hectare et des propriétés de mobilité et de persistance des produits permet de conserver un désherbage efficace tout en substituant les produits rémanents et polluants.

L'appareillage d'un site d'étude spécifique des transferts de désherbants appliqués en milieu urbain prévu dans le cadre du programme de la CORPEP en 1998 devrait permettre à terme d'ajuster les préconisations de désherbage pour ce secteur d'activité.

Parallèlement à cette action sur les pratiques et à la demande des élus, des sessions pratiques de formation à une utilisation raisonnée des désherbants ont été mises en place en collaboration avec le Centre National de Formation des Personnels Territoriaux (CNFPT). Cette formation dispensée par les centres de formation spécialisés et dont le contenu est proche de ceux mis en place pour la certification n'a pas reçu une reconnaissance d'équivalence.

Ces formations qui reçoivent un accueil très favorable seront progressivement étendues à l'ensemble des collectivités territoriales de Bretagne de même que la mise en place des plans de désherbage. Pour prolonger cette action de formation, un guide pratique de l'applicateur communal servant de support à ces formations va être édité en 5000 exemplaires. Un exemplaire sera transmis en fin d'année à chaque commune de Bretagne. La sensibilisation des élus est également prévue par le biais d'une plaquette qui sera tirée à 2000 exemplaires.

\section{Prise en compte des surfaces à risque}

\section{chez les particuliers}

La prise en compte de surfaces à risque chez les particuliers est apparue nécessaire au vu des résultats de suivi de la qualité des eaux mis en place sur les bassins versants du programme. Ces résultats mettent en évidence que les pratiques de désherbage des particuliers sont également associées à des contaminations des eaux superficielles. A cette fin, il a été décidé par les partenaires du programme de sensibiliser les particuliers par le biais d'un guide pratique destiné à leur proposer des solutions pour limiter ces pollutions.

Ce guide fait prendre conscience aux particuliers de l'existence d'aires à risque dans et aux abords de leurs propriétés (cours, terrasses, berges) sur lesquelles l'abandon des désherbants est de rigueur. Enfin pour limiter les risques de pollution liés au surdosage, des solutions pratiques sont proposées pour aboutir au dosage correct des désherbants.

Comme dans le cas des collectivités, un choix limité de substances actives prenant en compte les mêmes critères que précédemment est proposé pour les applications sur les zones à risque faible ou intermédiaire.

\section{Actions développées avec les administrations}

Concernant les administrations, des actions visant à améliorer leurs pratiques de désherbage ont également été entreprises. C'est avec la SNCF que les avancées apparaissent pour le moment les plus significatives. Les traitements herbicides mis en œuvre répondent à des besoins de sécurité par rapport à la signalisation automatique des voies et aux risques d'incendie pouvant se déclarer dans la végétation de bordure. Les désherbants totaux qui représentent actuellement la majorité des produits utilisés sont appliqués à l'aide de moyens techniques de pulvérisation très sophistiqués (trains désherbeurs) qui permettent, entre autres, de changer de produits sur des portions de voies présentant des contraintes particulières.

Cette opportunité a été saisie pour mettre en place une expérimentation visant à mesurer l'impact sur la qualité de l'eau, de la substitution des désherbants racinaires par des produits foliaires et antigerminatifs a priori moins polluants. Cet essai a été réalisé sur un bassin d'action renforcée où des pics de diuron ont été associés au passage du train désherbeur. L'expérimentation a consisté à substituer le diuron par une spécialité en cours d'homologation contenant du diflufénicanil et du glyphosate (le diflufénicanil est une substance a priori peu mobile). L'impact sur la qualité de l'eau a été vérifié par des mesures amont/aval de la zone traitée destinées à évaluer l'effet direct (pulvérisation dans les fossés circulants) et l'effet indirect (lié aux pertes de produit par ruissellement). L'efficacité du désherbage est mesurée au travers d'un suivi floristique.

Les premiers résultats des mesures sur la qualité des eaux sont encourageants puisqu'aucun des deux produits de substitution n'a été retrouvé dans les eaux. Sur le plan de l'efficacité herbicide, les notations floristiques font apparaittre une efficacité satisfaisante par rapport au niveau de sécurité souhaité par la SNCF.

Les résultats de cette expérimentation permettent d'envisager, dès que la spécialité sera homologuée, une modification des pratiques de désherbage des voies sur les bassins versants de BEP II concernés.

Concernant l'entretien des dépendances routières, placé sous la responsabilité des Directions Départementales de l'Equipement et des Conseils Généraux, les actions sont restées pour le moment limitées. 


\section{III - UNE BOÎTE À OUTILS QUI S'ENRI- CHIT POUR LES PARCELLES ET ZONES À RISQUE}

Le fondement des actions de réduction des pollutions diffuses reposant sur l'adaptation des itinéraires techniques de désherbage au niveau de risque des parcelles ou des zones à désherber, les études annuelles de la CORPEP ont été réorientées depuis le début du programme Bretagne Eau Pure vers la création ou la validation de programmes de désherbage efficaces et respectueux de la ressource en eau.

Nous présenteront ici les avancées significatives obtenues sur la réduction des transferts de produits par la mise en œuvre de programmes de substitution, de réduction des intrants, de travail réduit du sol et de l'aménagement du paysage. Enfin nous aborderons l'aspect réglementaire mis en place au plan local pour limiter la pollution des eaux superficielles par l'atrazine et le diuron.

\subsection{La substitution : validation par la CORPEP avant diffusion sur le terrain}

La substitution des substances actives les plus fréquemment associées à des dépassements des objectifs du programme BEP II est apparue comme un des premiers outils à mettre en œuvre sur les bassins étant donné une apparente facilité de réalisation et une relative acceptation par les acteurs de terrain. Mais avant de proposer ces modifications de pratiques aux distributeurs de produits (coopératives, négociants) intervenant sur les différents bassins versants, il a été nécessaire de valider ces nouveaux itinéraires de manière à garantir simultanément un résultat en termes de désherbage et d'amélioration de la qualité des eaux. Cette étape préalable, qui a pu être franchie grâce aux expérimentations conduites depuis 1996 dans le cadre des programmes annuels de la CORPEP, a permis de réaliser le classement des substances actives en trois groupes (Cf paragraphe 2.2.1).

Toujours concernant cette phase d'étude préalable au lancement des programmes de substitution sur le terrain, il faut mentionner également le travail de mise au point du dosage en routine dans les eaux des substances de remplacement. A ce niveau, il faut souligner la mise en place, dans le cadre de la CORPEP, d'un groupe de travail régional " laboratoires". $\mathrm{Ce}$ groupe qui rassemble tous les laboratoires régionaux pratiquant des dosages de pesticides dans les eaux a pour mission de mettre au point des méthodes de dosage de substances susceptibles d'être présentes dans les eaux et visà-vis desquelles au plan local nous sommes démunis. Il a également en charge l'organisation d'intercalibrations régionales qui font partie intégrante des programmes annuels de la CORPEP.

Actuellement, il est possible de suivre dans les eaux la totalité des molécules de substitution qui ont été retenues dans les chartes phytosanitaires.

Par ailleurs au plan régional, la surveillance de ces produits de substitution s'effectue sur le réseau des rivières de la CORPEP. Cette veille permet des réajustements rapides des préconisations dans les chartes.

\subsection{Désherbage mixte amélioré : efficace et non polluant}

Les premiers résultats obtenus en 1996 mettaient en évidence un double intérêt de cette technique associant au binage des inter-rangs un désherbage chimique sur le rang, à savoir retarder le déclenchement du ruissellement et diminuer de $60 \%$ les quantités de désherbants apportées à l'hectare $[2,3]$. Afin d'optimiser le transfert de la technique au terrain, on a décidé en 1997 de renforcer les études pour améliorer ses performances technico-économiques.

En 1997 quatre expérimentations ont été conduites simultanément sur le thème. L'une d'entre elles abordait l'aspect efficacité, une seconde concernait son coût et sa faisabilité en matière d'organisation de chantiers et les deux autres portaient sur la quantification des transferts de désherbants vers les eaux.

Les résultats de ces travaux permettent actuellement d'apporter des informations techniques et pratiques aux agriculteurs sur les différents points suivants.

Les machines (désherbineuses) qui leur étaient proposées sur le marché jusqu'en 1997 pouvaient être considérées comme des prototypes tant les systèmes de pulvérisation étaient rudimentaires et mal réglés. Ce défaut à lui seul aurait abouti au rejet définitif de la technique sur le terrain. Les améliorations ainsi que les réglages à apporter aux machines et aux systèmes de pulvérisation sont désormais consignés dans un cahier des charges qui a été remis aux différents constructeurs.

Des programmes de désherbage performants ont été élaborés à l'aide de machines correctement réglées et avec des produits de substitution amenant le coût total à l'hectare de la technique au même niveau que celui correspondant à un désherbage en plein pratiqué avec les molécules de références (atrazine, alachlore).

Vis-à-vis des transferts de produits vers les eaux, les résultats fournissent également des garanties d'efficacité sur la réduction des flux de pesticides qui est liée principalement à la réduction des quantités de substances actives apportées à I'hectare. Ces résultats sur les transferts ont également précisé les limites de cette technique par rapport au ruissellement. En effet, il apparaît clairement que le binage seul ne garantit pas une réduction des transferts car son effet est temporaire. L'érosion est au contraire favorisée par une relative fragilité des agrégats, ce qui peut dans certaines situations limiter la mise en œuvre de cette technique.

D'autres outils basés sur une simplification du travail du sol sont actuellement à l'étude sur un réseau de parcelles de références localisées dans des stations des Chambres départementales d'Agriculture et de I'INRA de Rennes.

Parmi ces outils, on citera le semis sous litière avec lequel on vise la valorisation des débris végétaux issus de la destruction du couvert végétal pour protéger la surface du sol de l'impact de la pluie et favoriser l'infiltration de l'eau dans le sol. Cette technique met en œuvre un semis direct de maïs à l'aide de machines ne travaillant le sol que sur la bande de semis (paraplow, combiplow) ce qui préserve dans les interrangs un mulch issu du couvert végétal précédent.

Les premiers résultats obtenus en 1998 laissent présager que la boîte à outils va continuer à s'enrichir.

\subsection{L'aménagement du paysage pour limiter l'érosion et les flux de pesticides}

Dans un bassin versant, certains éléments du paysage favorisent la circulation de l'eau (fossés, drains) ou au contraire la ralentissent (haies, talus, etc.). Certaines zones dites tampons possèdent un pouvoir de filtration et d'épuration de l'eau qui circule des parcelles vers le réseau hydrographique. Elles décantent les particules de sol érodé et 
retiennent puis dégradent les résidus de produits phytosanitaires. Le maintien ou la réhabilitation de zones tampons naturelles (haies, talus, roselières etc.) comme l'implantation de zones tampons artificielles (dispositifs enherbés) participent à la protection des ressources en eau et à la restauration de leur qualité en limitant la pollution diffuse.

Bien que la mise en place de ces dispositifs nécessite un engagement collectif impliquant la plupart des agriculteurs du bassin versant, ainsi que les gestionnaires de l'espace et de l'eau, il est également possible d'agir à l'échelle parcellaire en implantant sur les parcelles à risque élevé des bandes enherbées dont l'efficacité a été démontrée grâce aux études réalisées dans l'ouest de la France par l'Institut Technique des Céréales et des Fourrages.

Des travaux récents menés au sein de la CORPEP ont permis de vérifier que l'association de plusieurs de ces outils sur les parcelles à risque élevé de ruissellement assurait une protection quasi totale de la ressource en eau superficielle. C'est le cas par exemple de l'association bande enherbée et désherbage mixte.

\subsection{Un renforcement des mesures règlementaires au plan local}

Une étude portant sur l'évaluation du risque écotoxicologique réalisée par l'unité d'Ecotoxicologie aquatique de I'INRA de Rennes à partir des mesures de pesticides relevées dans les rivières bretonnes, a mis en évidence que, pour au moins trois substances (l'atrazine, le diuron et le lindane), le seuil de toxicité chronique directe est régulièrement dépassé.

Ce constat a amené la prise d'arrêtés préfectoraux dans les quatre départements bretons, limitant à partir du $1^{\text {er }}$ septembre 1998 les conditions d'emploi des deux premières substances citées, le lindane faisant l'objet d'un retrait d'homologation à partir du ler juillet 1998.

Dans le cas de l'atrazine, il convient de souligner la cohérence de cette mesure règlementaire avec les actions développées sur les bassins versants du programme Bretagne Eau Pure II. En effet, la restriction d'emploi ne concerne que les applications sur les parcelles situées en bordure de cours d'eau qu'elles soient drainées ou non.

Vis-à-vis du diuron, la mesure se traduit par une interdiction d'emploi pendant la période de l'année au cours de laquelle les conditions hydrologiques ne permettent pas une dilution suffisante de la substance active dans le milieu.

\subsection{Un manque d'information sur les pollutions ponctuelles}

Depuis son origine la CORPEP a mené des travaux uniquement sur la réduction des pollutions diffuses issues des parcelles ou des zones traitées. D'autres sources potentielles de pollution liées à de mauvaises pratiques survenant au moment de la mise en œuvre des traitements telles que l'élimination inadéquate des fonds de cuves, des eaux de rinçage sur des surfaces imperméables, ainsi qu'un stockage anarchique des produits n'ont pas été prises en compte. II ne faut pas perdre de vue en effet que quelques grammes de substance active suffisent à polluer l'eau de façon inacceptable. Par conséquent, tous les apports intempestifs de produits aux zones non ciblées (cours de ferme, de collectivités, chemin, fossé etc.) et à plus forte raison directement au milieu aquatique (ruisseau, mare, etc) doivent être évités impérativement.

L'importance de cette pollution ponctuelle par rapport à la pollution diffuse reste donc à quantifier. Ce travail, qui nécessite la mise en place d'expérimentations en conditions

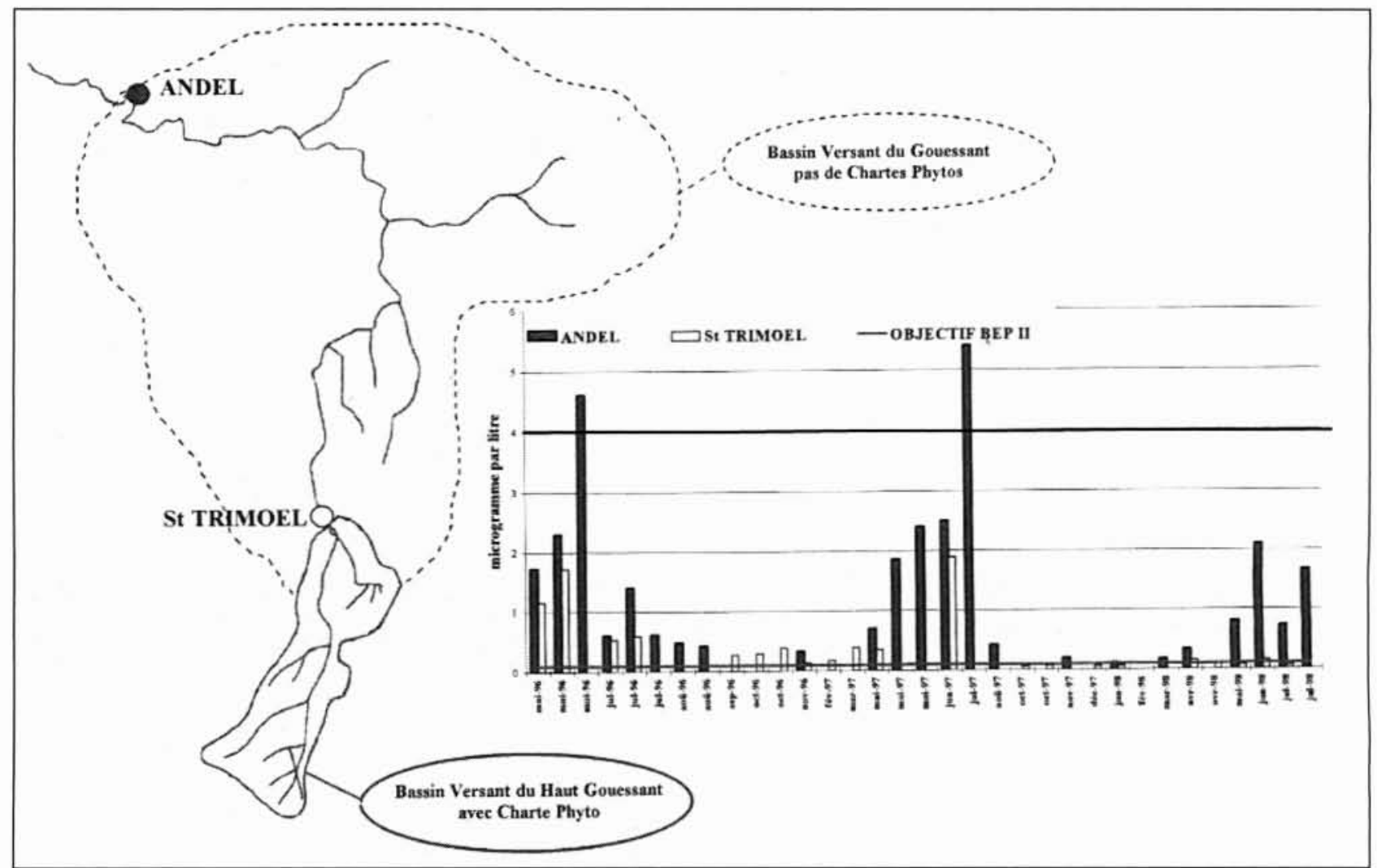

2. Evolution des concentrations en atrazine mesurées à la prise d'eau de Saint Trimoel et à Andel. 
contrôlées, constitue un véritable programme de recherche. II fait partie des objectifs prioritaires de la CORPEP pour les années à venir. Sans attendre les conclusions de ces travaux. il a été mis en place sur les bassins versants avec l'appui des firmes, des distributeurs et des techniciens des Chambres d'Agriculture, des campagnes de sensibilisation de tous les utilisateurs aux bonnes pratiques qui protègent l'eau.

\section{D DES PREMIERS RÉSULTATS SUR LA QUALITÉ DE L'EAU}

Cette démarche basée sur le repérage des sous-bassins à risque prolongée par le classement des parcelles et zones à risque sur lesquelles sont préconisés des itinéraires de désherbage non polluant consignés dans des chartes phytosanitaires fournit des résultats tangibles sur la qualité de l'eau au bout d'une année de mise en cuvre.

On observe actuellement sur deux bassins de démonstration sur lesquels elle a été appliquée dans son intégralité, une amélioration significative de la qualité des eaux brutes puisque les objectifs du programme BEP II ont été respectés depuis juillet 1997 vis-à-vis de l'atrazine. Cette substance active correspond probablement au cas le plus difficile à résoudre compte tenu de sa mobilité dans les sols et de sa persistance.

En se référant aux données de suivi de la figure 2 , il apparaît clairement que l'amélioration constatée sur le bassin de démonstration du Haut Gouessant est bien le résultat de l'application des chartes phytosanitaires puisqu'à l'aval du bassin (Andel), où ces chartes n'ont pas été mises en place, le profil d'évolution des concentrations reste identique à celui des années précédentes, de même que les niveaux de concentrations.

Les produits de substitution de l'atrazine, à savoir le nicosulfuron et la sulcotrione ont été recherchés à l'exutoire du bassin de démonstration mais n'ont jamais été détectés. Ces résultats sont confirmés par ailleurs depuis deux ans dans le cadre du réseau de surveillance régional de la CORPEP.

Enfin sur ce bassin, il convient de noter la persistance de deux problèmes de pollution : le premier lié aux désherbage des céréales (isoproturon, chlortoluron), le second en relation avec les pratiques de désherbage du secteur non agricole (diuron). Le premier s'explique logiquement étant donné l'absence de préconisation concernant les céréales dans la charte phytosanitaire. Le second correspond aux pratiques des particuliers qui restent les seuls utilisateurs de cette substance active sur le bassin. En effet la seule agglomération présente sur ce bassin a réalisé son plan de désherbage et substitué le diuron par des désherbants foliaires et anti-germinatifs de même que les autres utilisateurs potentiels pour l'entretien des dépendances routières.

Sur un autre bassin de démonstration où le classement des parcelles a été réalisé en totalité et prolongé par une charte phytosanitaire qui prend en compte les pratiques de désherbage du maïs et des céréales, les résultats tangibles qui ont été observés sur la qualité de l'eau concernent l'atrazine et les désherbants céréales.

Ces premiers résultats qui devront être confirmés à la fois dans le temps et l'espace démontrent que la reconquête de la qualité des eaux superficielles est possible vis-à-vis du paramètre pesticides à condition d'adapter les itinéraires de désherbage en fonction du risque des parcelles et des zones à désherber et de limiter les sources ponctuelles de pollution survenant au moment de la mise en ouvre des traitements.

\section{$V \square$ CONCLUSION}

La démonstration a été faite sur deux bassins du programme Bretagne Eau Pure II que la reconquête de la qualité de l'eau est possible vis-à-vis du paramètre pesticides. Ces résultats encourageants ne signifient pas pour autant que la bataille pour l'eau pure est définitivement gagnée. Il faut maintenant les pérenniser dans le temps et l'espace. Cette seconde étape s'annonce aussi difficile que la première étant donné que l'obtention de résultats significatifs sur la qualité de l'eau repose désormais sur l'adoption de ces nouvelles pratiques par tous les utilisateurs et prescripteurs intervenant sur les bassins versants.

Le travail de recherche appliquée doit également se poursuivre en amont pour continuer à servir les actions sur les bassins versants. De même, le maintien d'une passerelle entre les travaux de recherche appliquée et les actions développées sur les bassins versants permettra d'améliorer et d'ajuster rapidement si besoin la démarche mise en place pour reconquérir la qualité de l'eau.

\section{Bibliographie}

[1] Atrotisshat P., Souividant H., Bagti M. C.. Simon F., 1996, - Analyse des facteurs de risque de transferts de pesticides dans les paysages. Etablissement d'une hiérarchie de ces risques : application au calcul d'un indice de risque par bassin versant et par parcelle. Rapport final de la convention 95.00.023 avec I'Agence de I'Eau Loire Bretagne. Ecole Nationale Supérieure Agronomique de Rennes, Rennes. 22 p.

121 BRETAGNE EAU PURE, 1996. - Le bon usage des produits phytosanitaires, guide technique. L'exemple du maìs cultivé en Bretagne. SRPV. Rennes, $86 \mathrm{p}$

[3] BRETAGNE EAU PURE. 1995, - Qualité des eaux et produits phytosanitaires : du diagnostic à l'action. BEP. Rennes, $189 \mathrm{p}$.

141 CORPEN, 1996. - Qualité des eaux et produits phytosanitaires : proposition pour une démarche de diagnostic. $120 \mathrm{p}$.

[5] CORPEP. Décembre 1990. - Mise en place d'une cellule d'orientation régionale pour la protection des eaux contre les pesticides. Préfecture de la région Bretagne, Comité technique de l'eau. $15 \mathrm{p}+$ annexes.

|6| Gil..t H., Cifment M., Chossy A. M., Stux R., 1995. - Evaluation du niveau de contamination des eaux de surface par les produits phytosanitaires. J Eur Hydrol tome 26, fase 1.1995, p5 à 82.

171 Gilt.1 H., 1995. - Contamination des eaux superficielles de l'Ouest de la France par les produits phytosanitaires : du diagnostic à quelques propositions d'actions. ANPP. Seizième conférence du Columa, Journées internationales sur la lutte contre les mauvaises herbes. Reims 6,7,8 Décembre 1995.

[8] Gllı.1 H.. 1998. - Plan d'action pesticides pour la Bretagne. Préfecture de la Région Bretagne - Service Régional de la Protection des Végétaux, $20 \mathrm{p}+$ annexes.

[9] VAll.ANT M. et al, 1995. - A multicriteria estimation of the environnemental risk of chemicals with the SIRIS method. Toxicology mode ling. I (I), pp $57-72$. 